\title{
LA MICROSCOPIE DE PHOTOÉMISSION D’ÉLECTRONS, UN OUTIL MULTIDIMENSIONNEL POUR L'OPTIQUE CHAMP PROCHE
}

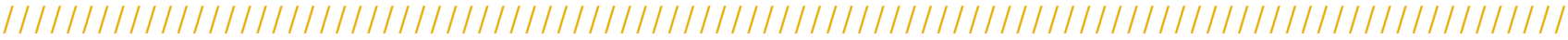

\section{Arthur LOSQUIN ${ }^{1}$, Ludovic DOUILLARD ${ }^{2 *}$}

${ }^{1}$ Thales Research and Technology, 1 avenue Augustin Fresnel, F-91767 Palaiseau, France

2 Univ. Paris-Saclay, CEA, CNRS, SPEC, F-91191 Gif-sur-Yvette, France

*ludovic.douillard@cea.fr

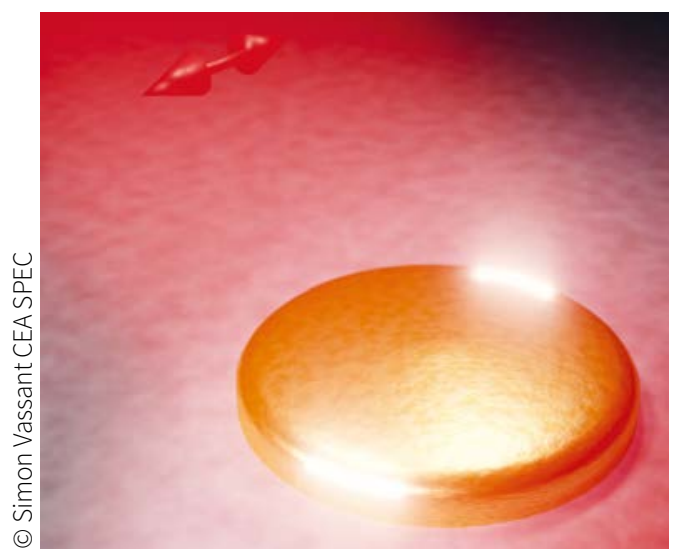

\begin{abstract}
La microscopie de photoémission d'électrons constitue une technique non intrusive d'investigation du champ proche optique. Son domaine d'application actuel autorise une approche physique multidimensionnelle des phénomènes d'optique de champ proche aux échelles spatiale ( $\mathrm{nm}$ ), temporelle (fs) et spectrale (meV).
\end{abstract}

\section{https://doi.org/10.1051/photon/202010235}

Article publié en accès libre sous les conditions définies par la licence Creative Commons Attribution License CC-BY (http://creativecommons.org/licenses/by/4.0), qui autorise sans restrictions l'utilisation, la diffusion, et la reproduction sur quelque support que ce soit, sous réserve de citation correcte de la publication originale.

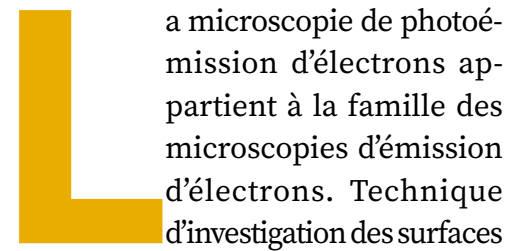
de la physique du solide, elle constitue aujourd'hui un outil d'investigation d'intérêt pour l'optique champ proche.

\section{PRINCIPE D'IMAGERIE \& CARACTÉRISTIQUES PRINCIPALES.}

Développée dès les années 1930 [1], la microscopie de photoémission d'électrons, connue sous l'acronyme anglosaxon PEEM pour PhotoEmission
Electron Microscopy, possède pour principe d'imagerie la collection des électrons émis par une surface par effet photoélectrique (phénomène physique d'émission d'un électron d'un solide par absorption d'un, voire de plusieurs photons). Il s'agit d'une microscopie de type plein champ, dontle principe de construction de l'image est équivalent à celui mis en œuvre dans un microscope optique. Sa résolution spatiale peut atteindre la dizaine de nanomètres, voire $1 \mathrm{~nm}$ sur des instruments corrigés des aberrations des optiques électroniques.

Dans sa configuration classique, l'échantillon est illuminé par un faisceau de lumière dans la gamme des longueurs d'onde UV (lampe à vapeur de mercure), voire $\mathrm{X}$ (source $\mathrm{X}$, radiation synchrotron). L'image obtenue possède un contraste fonction de l'énergie cinétique des électrons collectés et reflète directement le travail de sortie du matériau ou la distribution des espèces chimiques présentes en surface. A l'instar de la microscopie électronique à balayage, la microscopie PEEM constitue alors un outil d'investigation des surfaces, particulièrement adapté à la conduite d'études spatio-temporelles de croissance de matériaux et de suivi de réactions de surface. 
Par substitution de la source de lumière UV/X par une source laser femtoseconde opérant dans le domaine des longueurs d'onde visibles/ IR, la microscopie PEEM devient une méthode non-intrusive de cartographie du champ proche optique. Sur un plan physique, les phénomènes d'exaltation du champ proche optique omniprésents en nanophotonique et plasmonique magnifient l'effet photoélectrique. Compte tenu des énergies de photons mises en jeu au regard de l'énergie minimum requise pour extraire un électron d'un solide (travail de sortie) des matériaux étudiés, les électrons collectés résultent d'un processus de photoémission non-linéaire, $c f$. fig. 1. Les images obtenues affichent alors un fort contraste reflétant les zones d'exaltation du champ proche. Corollaire instrumental, l'emploi d'une source laser impulsionnelle cohérente dote la microscopie PEEM du large éventail d'analyse des spectrométries optiques (longueur d'onde, polarisation, profil temporel) et autorise l'investigation multidimensionnelle des phénomènes d'optique en champ proche aux échelles multiples: spatiale (nm), temporelle (fs) et spectrale (meV).

\section{APPLICATIONS}

Cartographie \& Manipulation du champ proche optique. En raison des multiples degrés de liberté offerts par une impulsion laser (polarisation, longueur d'onde, profil temporel), la microscopie PEEM constitue un puissant outil de cartographie, diagnostic nanométrique et manipulation du champ proche optique.

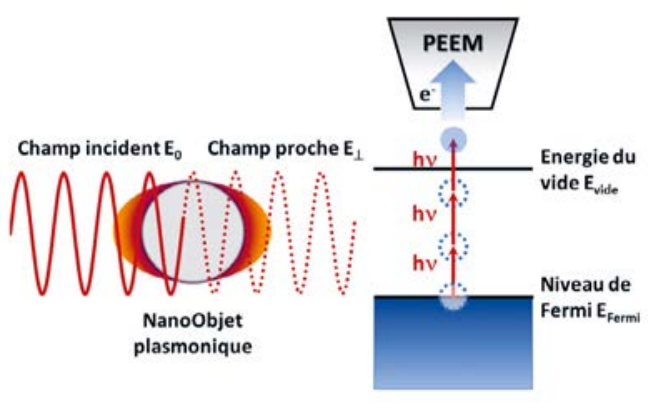

Figure 1.

Principe d'imagerie de la microscopie PEEM. Emission d'électrons par effet photoélectrique non linéaire dans le champ proche d'un nano-objet métallique à résonance plasmon.

La figure 2 illustre ces possibilités dans le cas de résonances plasmons localisées sur des objets métalliques de dimensions sub-longueur d'onde. Le premier objet considéré est une sphère Au hérissée de multiples pointes obtenue par synthèse colloïdale, fig. 2.1.(a). L'emploi d'une source de lumière polarisée autorise l'excitation sélective d'une pointe quelconque de lobjet par alignement du champ électrique incident sur son axe de symétrie, fig. 2.1 (c, d, e). Compte tenu de sa géométrie, l'excitation d'une pointe se manifeste

Figure 2.

Imagerie PEEM - Cartographie \& Manipulation du champ proche optique

1. Par le biais de la polarisation. Excitation $800 \mathrm{~nm}$, polarisations variables, nanoétoile Au, barre d'échelle $100 \mathrm{~nm}$. Imageries MEB (a), PEEM (b,c,d,e). Adapté de [2],

2. Par impulsions croisées décalées en temps. Excitation $800 \mathrm{~nm}$, polarisations croisées, nœud papillon Au, barre d'échelle 200 nm. Adapté de [3]. par l'apparition d'un point chaud du champ proche optique à son extrémité. Le diamètre d'un point chaud quantifie la résolution optique de l'instrument, en loccurrence environ $25 \mathrm{~nm}$. Le facteur d'exaltation de l'amplitude du champ électrique est de l'ordre de quelques dizaines. Cette méthode d'excitation sélective d'un état propre d'une résonance plasmon par le biais de la polarisation constitue une technique simple de manipulation du champ proche optique aux petites échelles [2].

Une seconde voie d'ingénierie du champ proche consiste à employer des impulsions croisées décalées en temps pour manipuler des interférences inter-modales à l'échelle locale [3]. Le degré de liberté physique mis à profit est ici la phase relative des impulsions. La figure 2.2 démontre la réalisation d'une telle approche sur un objet de type nœud papillon («bowtie») fabriqué par lithographie électronique. Le jeu des interférences inter-modales autorise un contrôle précis des positions des points chauds à la surface de lobjet.

Dynamique plasmonique. En parallèle de ces aspects cartographiques, la microscopie PEEM est également adaptée à l'étude des dynamiques spatio-temporelles plasmoniques se manifestant à la fois à des échelles nanométrique etfemtoseconde. La méthode couramment adoptée consiste à utiliser deux impulsions femtosecondes identiques retardées l'une de l'autre par le biais d'un interféromètre à ligne à retard. Le contraste PEEM change alors en fonction du retard entre les deux impulsions d'une façon qui reflète la dynamique plasmonique.
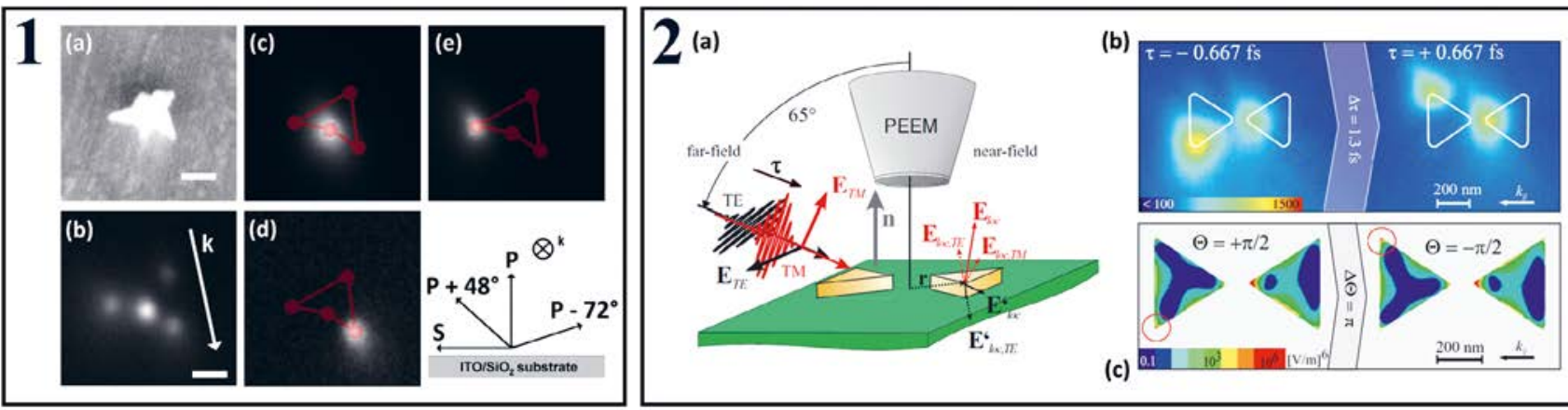

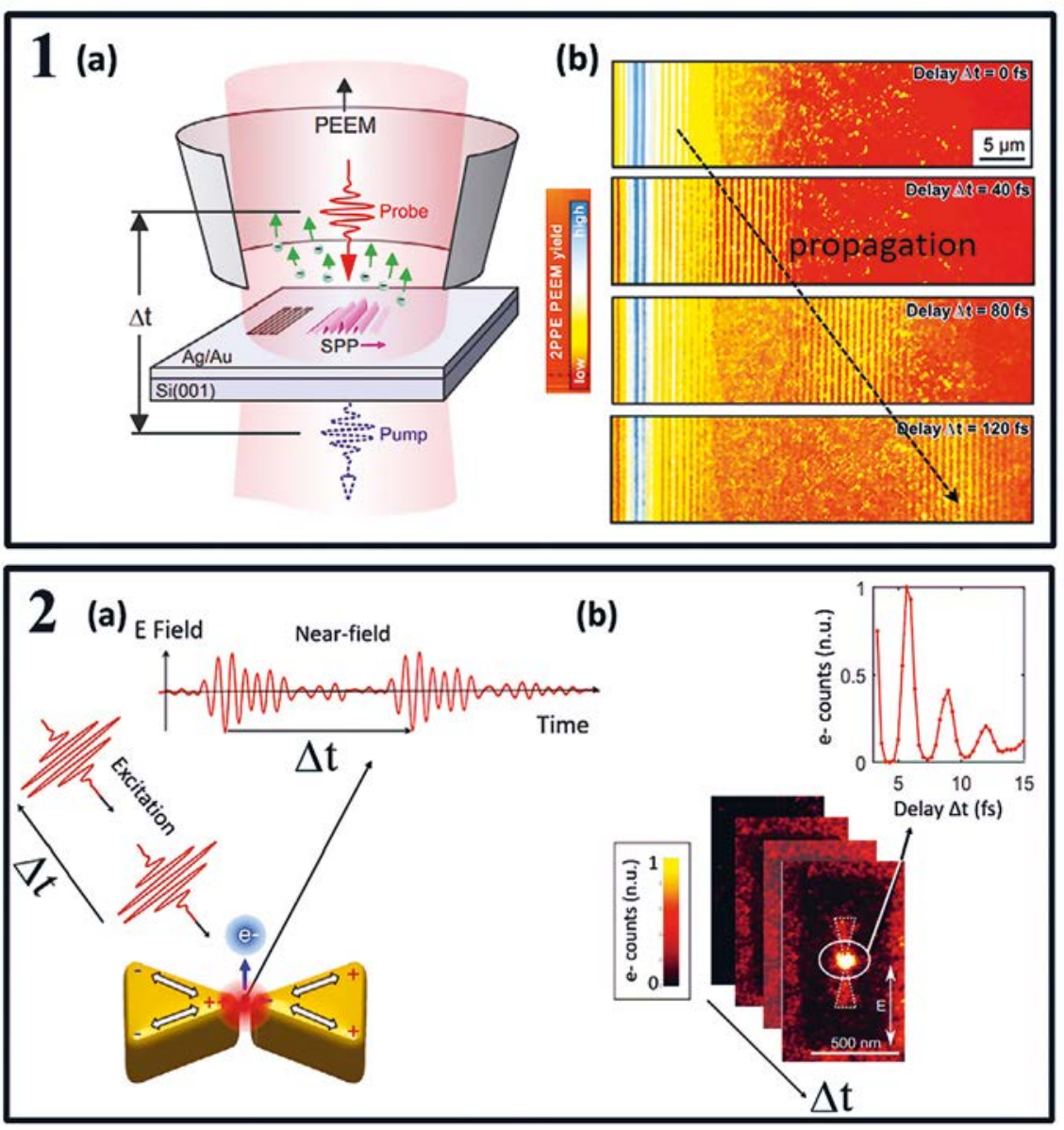

Figure 3.

Étude de dynamiques plasmoniques par microscopie PEEM résolue en temps

1. Étude PEEM de la propagation de paquets d'onde plasmoniques à la surface d'un film métallique monocristallin Au. Adapté de [4],

2. Étude PEEM de la dynamique d'oscillation de paquets d'onde plasmoniques à la surface d'une nanoantenne «bowtie » Au. Adapté de [5].

Une première approche de ce type, conduite sur un film Au monocristallin, est rapportée figure 3.1 [4]. Par illumination d'un réseau de couplage, la première impulsion («pompe») génère des paquets d'ondes de plasmons de surface polaritons. La deuxième impulsion (« sonde ») interfère localement avec ces paquets d'onde. L'interférence constructive entre un paquet d'onde et l'impulsion sonde se traduit par une exaltation de la photoémission à la position du paquet d'onde. Ainsi, les images PEEM obtenues pour différents retards entre impulsions visualisent directement la propagation du paquet d'onde et permettent d'accéder à ses vitesses de phase et de groupe.

Un second exemple illustré sur la figure 3.2 porte sur la dynamique d'oscillation de paquets d'onde plasmoniques à la surface de nanostructures métalliques, nanoantennes «bowtie » $\mathrm{Au}[5]$. Dans ce scénario, chacune des deux impulsions excite un paquet d'ondes, dont la dynamique d'oscillation est dictée par les modes propres plasmons de la nanostructure. Les interférences entre impulsions conduisent à des oscillations du signal PEEM pour des retards inférieurs à quelques dizaines de femtosecondes. Ces oscillations renseignent sur la dynamique du paquet d'onde, notamment sur sa fréquence d'oscillation, de façon locale. Ici, la dynamique est accessible dans l'interstice de la nanoantenne par un effet d'exaltation. A noter que cette méthode nécessite des durées d'impulsions de l'ordre du temps de vie des modes propres plasmoniques ( 5 fs dans le travail considéré).

\section{Wavefront sensors and \\ adaptive optics for \\ optical metrology, \\ lasers and microscopy}

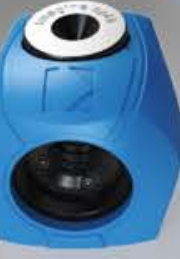

Optical metrology applications
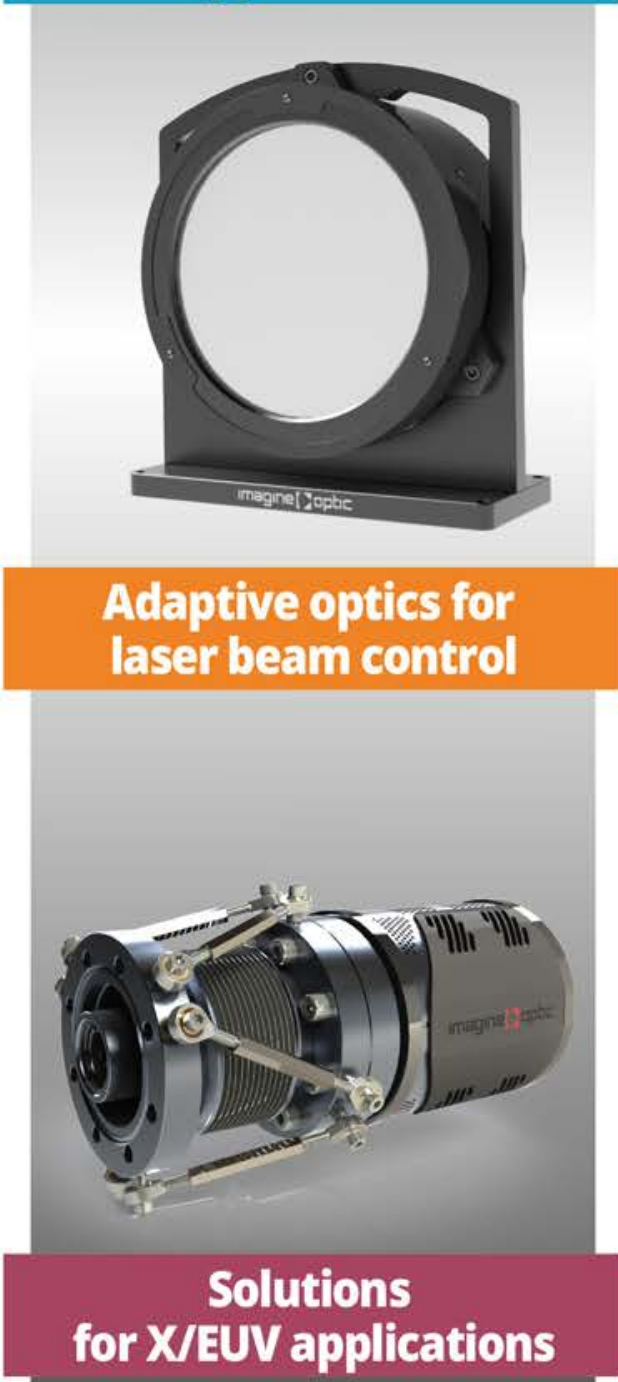

Contact us for more details: contact@imagine-optic.com or +33164861560 

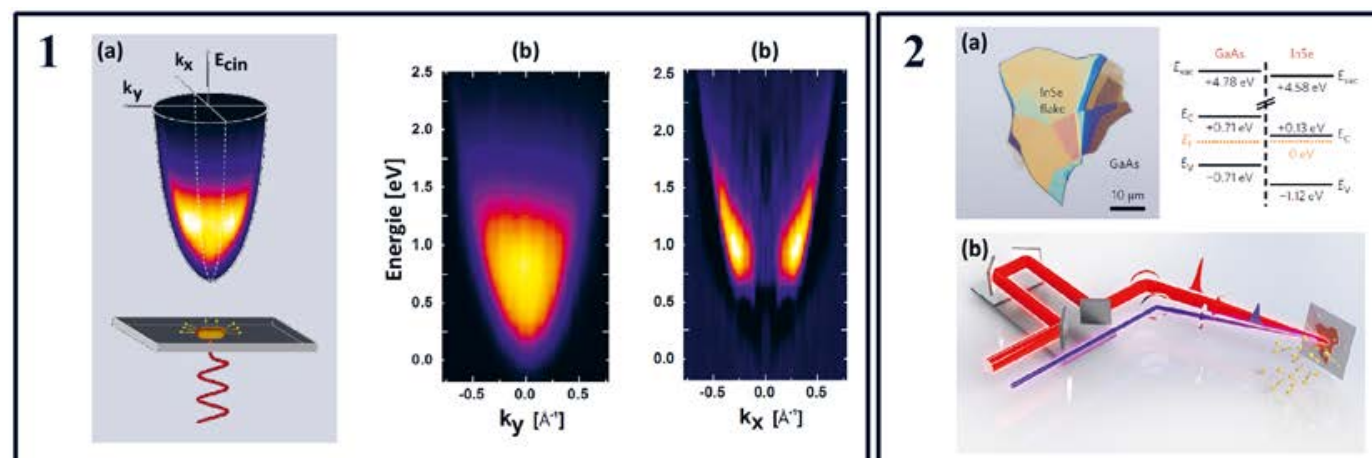

(b)
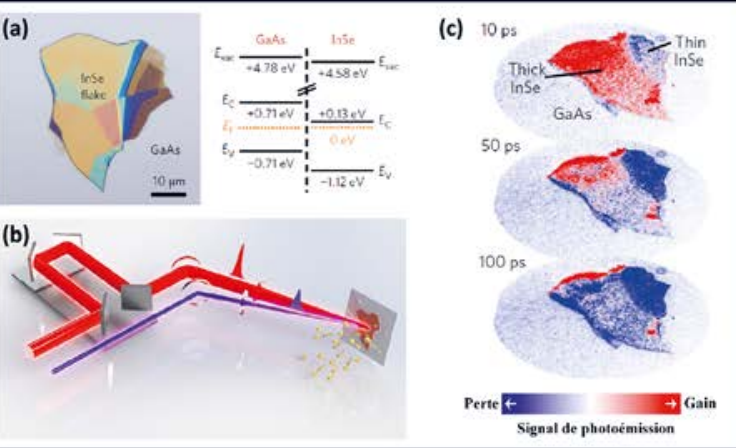

Perspectives. En parallèle des aspects d'optique de champ proche, la nature même de son signal ouvre à la microscopie PEEM de nouveaux axes de recherche. Elle se révèle ainsi un outil particulièrement adapté à l'étude de la physique des électrons chauds, éléments clefs de thématiques actuelles telles que la photochimie, la photo- et l'électro- catalyses plasmoniques sur particules et/ou surfaces, la conversion d'énergie solaire, la production et le transport d'électrons aux petites échelles. Sur un plan instrumental, les perspectives visent à exploiter plus avant le caractère multiéchelle (nm, fs, meV) de la méthode. Les améliorations techniques portent à la fois sur les instruments (correction des aberrations des optiques, types de détecteurs d'électrons : à temps de vol, en spin) et les sources de lumière (sources ultrabrèves : femto ou atto seconde).

Une première illustration de ces tendances est rapportée figure 4.1. Létude en question porte sur le suivi, dans l'espace réciproque, de la distribution en moments et en énergie des électrons émis par un unique bâtonnet d'or à la résonance plasmon [6]. Elle est conduite sur un instrument à détection d'électrons par temps de vol. L'examen de la distribution des électrons révèle la compétition de deux processus d'émission électronique distincts. Le premier est un processus de nature multiphotonique, le second relève d'un processus par émission de champ, c'est-à-dire l'émission d'électrons par effet tunnel au travers de la barrière de potentiel de surface. Le passage de l'un à l'autre est piloté par lirradiance incidente. Ce type d'étude

Figure 4.

Applications multiéchelles ( $\mathrm{nm}$, fs, meV) de la microscopie PEEM pour l'étude de la physique d'électrons chauds.

1. Étude de la distribution en moment et en énergie des électrons émis par un unique bâtonnet Au à résonance plasmon. Adapté de [6].

2. Étude de la dynamique spatio-temporelle résolue en énergie du transport d'électrons dans une hétérojonction PN modèle GaAs / InSe. Adapté de [7].

illustre l'intérêt de la technique pour l'investigation des champs électromagnétiques de hautes intensités et son cortège de phénomènes physiques associés, tels que les effets pondéromoteurs, responsables de l'accélération des électrons dans le vide par le champ électrique de l'impulsion laser ou encore l'ionisation au-dessus du seuil (above threshold ionization ATI), un processus d'émission électronique par absorption d'un nombre inhabituellement élevé de photons.

Le second et dernier exemple porte sur le suivi par PEEM de la dynamique spatio-temporelle résolue en énergie du transport d'électrons au sein d'une hétérojonction photovoltaïque GaAs / InSe, fig. 4.2 [7]. L'approche adoptée est de type « pompe sonde » à deux couleurs : une première impulsion de longueur d'onde $800 \mathrm{~nm}$ génère la population délectrons chauds, suivie d'une seconde impulsion de longueur d'onde $266 \mathrm{~nm}$ qui éjecte cette population de la jonction. En analysant la répartition spatiale des électrons photoémis en fonction de leur énergie et du retard entre impulsions, on peut suivre le transport de la population d'un matériau à l'autre. Cette preuve de principe, obtenue sur une jonction PN modèle, illustre la pertinence de la microscopie PEEM pour revisiter certains phénomènes physiques macroscopiques bien connus par le biais d'une approche mésoscopique novatrice.

\section{CONCLUSION}

Aux côtés de techniques telles que les microscopies de champ proche optique à balayage de sonde (SNOM scanning nearfield optical microscopy), la microscopie de photoémission d'électrons PEEM constitue l'une des rares techniques de cartographie du champ proche optique aux petites échelles spatiales (nm). Issue des domaines de loptique et de la physique du solide, elle permet d'investiguer les phénomènes physiques aux petites échelles spatiales (nm), temporelle (fs) et spectrale (meV).

RÉFÉRENCES

[1] E. Brüche, Z. Phys. 86, 448 (1933)

[2] C. Hrelescu et al., Nano Lett. 11, 402 (2011)

[3] P. Melchior et al., Phys. Rev. B 83, 235407 (2011)

[4] P. Kahl, et al., Plasmonics 13, 239 (2018)

[5] E. Lorek et al., Opt. Express 23, 31460 (2015)

[6] M. Lehr, et al., Nano Lett. 17, 6606 (2017)

[7] M. Man, et al., Nature Nanotech. 12, 36 (2017) 